\title{
Analysis on Life Cycle of Network Public Opinion
}

\author{
J. QIAO, Z.H. GAO, Y.R. HUANG, Y.Y. HOU \& X.C. WEI \\ Beijing Institute of Science and Technology Information, Beijing, China
}

\begin{abstract}
As the rapid development of network throughout the world, network media has been recognized by the public as the "4th media" following newspaper, radio broadcast and television. Network has become one of the major carriers reflecting the social public opinion. In the communication framework, the network public opinion space is called by many domestic scholars as the network public opinion environment. This shows that both generation and development of network public opinion will invariably appear, develop and realize in a certain time and space environment, i.e a life cycle for network public opinion from starting to declining.
\end{abstract}

KEYWORD: network public opinion; Life cycle; H7N9 bird flue; Haze

\section{INTRODUCTION}

Network public opinion refers to popular online public opinion on different perspectives about social issues, it is an expressive form of public opinion, reflects influential and tendentious remarks and perspectives of the public against some hot issues and focus problems spread through Internet.

Network public opinion refers to social and political attitudes, beliefs and values held by the public against public issues and social managers around the occurrence, development and changes of intermediary social events, network public opinion is spread through network within a certain social space. Network public opinion reflects all the beliefs, attitudes, opinions and emotions expressed by more citizens against various types of phenomenon and problems in the community.

Formation of network public opinion is often very quick, a hot event plus an emotional opinion can become a fuse igniting public opinion throughout a wide range. On the one hand, when an event happens, the network users can immediately express their opinions online, while individual opinions can be quickly gathered as public opinions. On the other hand, opinions from different channels can interact mutually, online and offline opinions affect each other, thus to rapidly form a strong opinion momentum and create a huge impact on the community.

\section{LIFE CYCLE OF NETWORK PUBLIC OPINION}

For the life cycle of network public opinion, many scholars have developed its characteristics in different stages from the development history and backgrounds of the life cycle theory. Gu Mingyi believes that network public opinion can be divided into 5 stages, i.e the early propagation stage, the social informed stage, the social expression stage, social action stage and media memorial stage. Xie Kefan also divided network public opinion into five stages, i.e the potential stage, the incubation stage, the acceleration stage, the mature stage and the declining stage. While Wang Kequan believes that network public opinion will evolve into a development process consisting formation, high volatility, fluctuation and final dilution. Cao Jinsong believes that the development of network public opinion has to experience 4 stages (spread-gatheringdiscussion-popular) and 3 crossings (outburst, sublimation and continuation). Pan Chongxia divided the evolution of network public opinion into 3 stages: initial spread, rapid diffusion and slowly vanishing. Lan Yuexin and Deng Xinyuan divided network public opinion into occurrence stage, spreading stage and stabilizing stage. Wangqing, et.al divide the life cycle of network public opinion to the specific stages of incubation stage, appearance and outbreak stage, continuous evolution stage, recession remission stage and disappearance stage. 
Through study and analysis, Zhang Heng concludes the two meanings of the life cycle of network public opinion. (A) The life cycle of a network public opinion generated around a network event is just like a life, it will also experience a "life cycle" from starting to declining; (B) We can divide the network public opinion reflected through a network event into 4 stages, including the incubation stage, growth stage, mature stage and declining stage. Each stage has the following characteristics:

(1) Incubation stage

In this stage, most public opinion events appearing in the network are social issues concerned by network users, who express their positive or negative opinions online. These public opinion events slowly brew in this stage and stand out once sufficient strength is accumulated.

(2) Growth stage
When breaks out in the incubation stage, the network public opinion event will cause an uproar in the network, it attracts concerns from not only the network users, but also social media, a widespread concern will be attached in the community.

(3) Mature stage

Most network users form the common opinion, such a mainstream opinion symbolizes that the event reaches the culminate in this stage.

(4) Declining stage

The network public opinion event is settled, yet this does not mean the end of the event, it is only because that the concern of network users and media is attracted by new network events, the network users begin to focus on new objects, new events and new topics.

Through the study results mentioned above, the paper compares and summaries the characteristics of network public opinion in different stages.

Table 1. Table about comparison between characteristics of network public opinion in different sages

\begin{tabular}{|l|l|l|l|l|l|}
\hline Stage & Cause significance & Network popularity & $\begin{array}{l}\text { Concentration of } \\
\text { network opinions }\end{array}$ & Network energy & $\begin{array}{l}\text { Emergency } \\
\text { Progress }\end{array}$ \\
\hline Incubation stage & Fuzzy & Very low & Dispersed & Very weak & Begin to appear \\
\hline Growth stage & Increasingly prominent & Increased stably & $\begin{array}{l}\text { Gathering from } \\
\text { different channels }\end{array}$ & Gathering slowly & Develops slowly \\
\hline Mature stage & Completely prominent & Increased rapidly & Rapid concentration & Gathering rapidly & Develops rapidly \\
\hline Declining stage & Gradually eliminated & Declining gradually & Gradually dispersed & Declining gradually & Solved gradually \\
\hline
\end{tabular}

\section{ANALYSIS ON LIFE CYCLE OF NETWORK PUBLIC OPINION EVENTS}

Based on the top 10 news events and network public opinion hot events in 2013, some events are selected to perform analysis on life cycle. From the event selected, we can observe that network public opinion events are broadly divided into two types, including unexpected events, such as Ya'an earthquake, Hengda wins the champion, etc and continuing events, such as fog and haze weather, Chang E III, etc.

Table 2. List of network public opinion events

\begin{tabular}{|l|l|}
\hline H7N9 bird flu & Fog and haze \\
\hline Ya'an earthquake & Hengda wins the champion \\
\hline Salt seizing event & Rubber duck to China \\
\hline Dead pigs in Jiaxing & Mayun resigns from CEO \\
\hline Rare hot weather & Change E III \\
\hline Final appeal against Li** & Chen Yongzhou case \\
\hline Cancer village & Moyan wining the Nobel prize \\
\hline $\begin{array}{l}\text { Establishment of Shanghai } \\
\text { FTA }\end{array}$ & Shenzhou 10 \\
\hline $\begin{array}{l}\text { Opening of Beijing Garden } \\
\text { Exop }\end{array}$ & Third plenary session of 18 \\
\hline Taobao double eleven & Guo Meimei event \\
\hline
\end{tabular}

Two network public opinion events with different nature are selected from the above list for analysis.

\subsection{Analysis on life cycle of H7N9 bird flu event}

In the first half of 2013, "H7N9" became a popular word in the network. H7N9 subtype avian influenza virus is a type of influenza. In the end of March 2013, 3 patients infected by H7N9 bird flue were first found in Shanghai and Anhui. In April 2013, H7N9 bird flu began to outbreak throughout the state.

As a hot event in the network public opinion, the life cycle of the spread of H7N9 bird flue can be divided into 4 stages, as shown below:

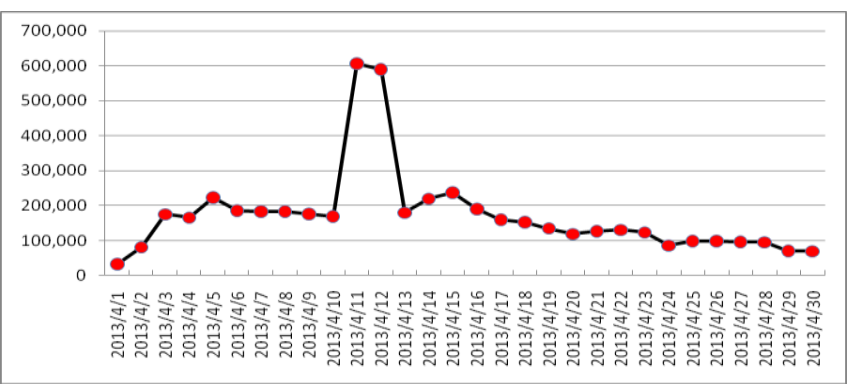

Figure 1. Schematic diagram about network communication life cycle of H7N9 bird flue 
It is shown in the life cycle curves of H7N9 bird flu event that it is formed by a single life cycle, thus it is a single-peak life cycle, such a life cycle has 4 obvious stages, particularly the mature stage. When entering the mature stage, such a single-peak curve will not form a relatively stable flat stage, instead, it will soon shows a rapid declining tendency after reaching the peak. The rapidness and openness of network communication make it impossible for the whole event to be spread in an objective environment, thus the concern about the event fluctuates seriously, then the event is likely to be understood in a wrong direction, this reflects the abruptness feature of network communication, which can not maintain the stability of the propagation environment and is not conductive to social stability.

Assuming to, in the incubation stage when the event starts initially, implement in-depth investigation against development of the epidemic situation, popularize relevant knowledge to the public; in the growth stage, take more stringent measures to prevent expansion of the epidemic and reduce panic of people, strengthen scientific propaganda and propose precautionary approaches; in the mature stage, when the event reaches the peak, any measure taken in this stage will increase people's panic and social unrest, therefore, we must give early warning and make relevant preparations in the growth stage, then the event would not get into an uncontrolled situation.

\subsection{Analysis on life cycle of haze event}

In 2013, "haze" became one of the annual keywords. The 4 haze processes in January of this year shrouded more than 30 provinces (autonomous regions and municipalities), in Beijing, only 5 days were not invaded by frog and haze. It is reported that, among the 500 largest cities in China, only less than $1 \%$ cities meet the air quality standard recommended by the World Health Organization, at the same time, among the 10 most polluted cities in the world, 7 cities are in China.

It is shown in the picture that the life cycle of the network public opinion event is composed of multiple life cycles, concern of people on the event lasted for a relatively long period. With the fluctuations of concerns on the event, the network public opinion event shows a wavy development trend, the curve peak is the highest point of concern by the public, while the lowest point in the curve represents that the concern by the public for the event reaches the lowest point, although the 4 stages in the life cycle of the event still exist, it will always repeat from the incubation stage to the declining stage, no significant fluctuation will appear. Such a life cycle with fluctuation enables the event to develop healthy in an objective direction.

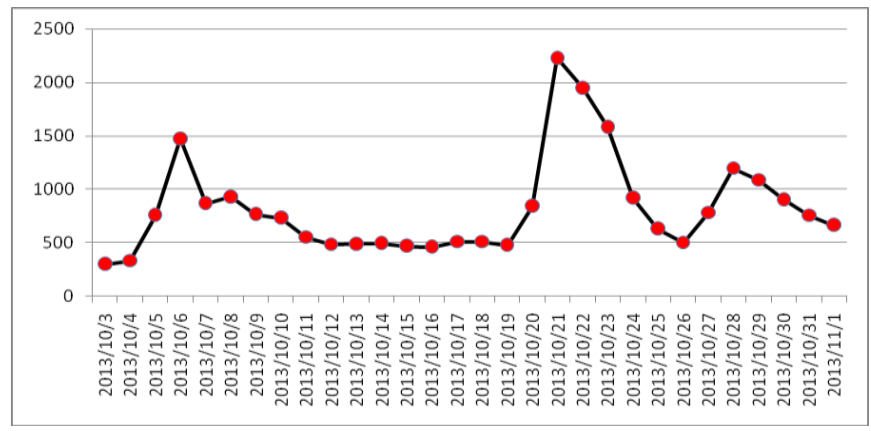

Figure 2. Schematic diagram about network communication life cycle of haze event

\section{CONCLUSION}

As the continuous innovation in network technology, network public opinion begin to be spread in multiple communication ways. To observe from the above two cases, the growth stage of network public opinion is the necessary way for development for network public opinion, how to avoid significant fluctuations in the event and give early warning in the growth stage is a critical step to prevent further expansion of the event.

Building a harmonious network environment is an important part in building a harmonious community, how to give full play to the internet in expression of public opinion and effectively control and eliminate its adverse influence has important social significance. Monitoring the life cycle of network public opinion and provide proper guidance is conductive to control the events development, only when we properly use the features of network communication, could we promote the health and stability of the communication environment.

\section{REFERENCES}

[1] Gu Mingyi, Zhou Renwei, Network public opinion and social network information transmission mode, Journalism \& Communication, 2009(5); 67-73

[2] Xie Kefan, Chen Gang, Cai Wenjing. Study on life cycle principle and cluster decision in network public opinion emergencies, Journal of Wuhan University of Technology (Social Science edition), 2010 (4): 482-486

[3] Wang Kequn. Analysis and response to network public opinion, Changbai Journal, 2010 (1): 90-93

[4] Cao Jinsong, Development law of network public opinion, News and writing, 2010 (5): 45-47

[5] Pan Chongxia. Analysis on evolution stages of network public opinion, Computer and Modernization, 2011(10).

[6] Lan Yuexin, Deng Xinyuan. Study on evolution rules of network public opinion emergencies. Journal of Information. 2011(08).

[7] Wang Qing, Cheng Ying, Chao Naipeng. Study on construction of network public opinion monitoring and warning indicator system, Library and Information Service, 2011(08) 